\title{
Análisis del impacto y actualización hasta 2018 del valor económico y social del Plan MAGNA de cartografía geológica a escala 1:50.000
}

\author{
Alfonso Herrero de Egaña(1), José Vivancos ${ }^{(2)}$, Ángel García-Cortés ${ }^{(3)}$ \\ (1) UNED. Facultad de Ciencias Económicas y Empresariales. Departamento de Economía Aplicada y Estadística. \\ Despacho 3.12. Paseo del Rey, 11. 28040 Madrid. \\ alherrero@cee.uned.es \\ (2) UNED. Facultad de Ciencias Económicas y Empresariales. Doctorando del Programa Economía y Empresa. \\ Paseo Senda del Rey, 112040 Madrid. \\ jvivancos7@alumno.uned.es \\ (3) Instituto Geológico y Minero de España. Ríos Rosas, 23. 28003 Madrid. \\ garcia.cortes@igme.es
}

\begin{abstract}
RESUMEN
En un trabajo publicado en 2005, García-Cortés y colaboradores realizaron una evaluación del valor económico y social del Plan MAGNA (García-Cortés et al, 2005a) en base al valor que las empresas usuarias de mapas geológicos daban a este producto, a su disposición al pago por estos mapas y al coste de la inversión geológica sustitutiva necesaria, en caso de su no existencia. El presente estudio parte de este precedente, en primer lugar, analizando el impacto de la difusión en publicaciones científicas de aquel estudio, y en segundo lugar, actualizando el valor económico y social hasta 2018 del Plan MAGNA de cartografía geológica de España a escala 1:50.000. En el presente trabajo se aplica la metodología desarrollada en 2005 a los nuevos datos disponibles entre 2004 y 2018 , con el objeto de obtener el valor actualizado de las ventas acumuladas de hojas MAGNA a empresas. Este nuevo proceso de evaluación ha permitido asimismo destacar varios puntos adicionales de interés, como los distintos perfiles de demanda correspondientes para cada diferente tipo de usuario de mapas geológicos en España, así como sobre la excelente rentabilidad obtenida por la inversión pública en dicho Plan MAGNA, gracias a los sustanciales ahorros generados a los distintos usuarios de dichos mapas en el desarrollo de sus actividades económicas, y finalmente sobre el impacto potencial de la actual descarga electrónica gratuita de las hojas geológicas del Instituto Geológico y Minero de España en su labor de diseminación del conocimiento geológico.
\end{abstract}

Palabras clave: análisis coste beneficio, descarga electrónica gratuita, divulgación científica, evaluación económica, mapa geológico.

\section{Impact analysis and update to 2018 of the economic and social value of the MAGNA Plan (geological mapping at 1:50,000 scale)}

\begin{abstract}
In a study published in 2005, García-Cortés and colleagues developed a social and economic evaluation for the MAGNA Plan (García-Cortés et al, 2005a) based on the value that user companies assigned to this product, their willingness to pay for these maps and if they are currently not available their willingness to pay for the necessary costs for the geological investment. This new study is based on the above precedent, first by analyzing the impact of its dissemination in scientific publications, and second by updating up to 2018 the economic and social value of the MAGNA Plan (Geological Map of Spain at 1:50,000 scale). The authors have applied the methodology developed in 2005 to the new data available from 2004 to 2018, in order to be able to update the value of the cumulative number of MAGNA geological survey sheets sold to companies. The new evaluation process has also allowed us to highlight some additional issues of interest, such as the different demand profiles for each of the various user types of geological maps in Spain and the excellent economic returns achieved by the Spanish public investment in the MAGNA Plan, thanks to the substantial savings generated by the different users of the maps in the development of their business activities, and finally regarding the potential impact of the current IGME (Spanish Geological Survey) free electronic downloading of the scientific dissemination of geological knowledge.
\end{abstract}

Keywords: cost/benefit analysis, free electronic downloading, economic valuation, geological map, scientific dissemination. 
Alfonso Herrero de Egaña, et al., 2020. Análisis del impacto y actualización hasta 2018... Boletín Geológico y Minero, 131 (4): $817-829$

\section{ABRIDGED ENGLISH VERSION}

\section{Introduction and methodology}

The main aim of this study is the updating of the previous calculation of the economic and social value of the MAGNA Plan geological 1:50,000 scale survey, developed by some of the authors for the occasion of the practical completion by the geological Survey of Spain (IGME) of the 2005 programme. The previous report was first published in the Boletín Geológico y Minero (García-Cortés et al., 2005a) followed by an additional bilingual Spanish-English diffusion specific publication (García-Cortés et al., 2005b), considering the sales data for the geological maps available up to 2003. At the time it was considered of interest to economically assess both the sales and the uses of the geological sheets, first as a measure for the suitability of the products offered in the IGME MAGNA Plan, but also for the potential need for any updates, additional developments or amendments, in order for IGME not only to more adequately fulfil but also to understand in detail the very specific requirements of the different users of this geological cartography.

During a preliminary bibliographic review, carried out before addressing the valuation updating objective sought, the authors were very positively surprised by the discovery of the substantial scientific impact of the previous publications on the monetary valuation method of the geological 1: 50,000 cartography of the MAGNA Plan, a measure for its social value. Figure 1 shows the evolution of the number of citations of these publications for the 2005-2018 period. The time evolution of the citations received by both publications by Garcia-Cortés et al. (2005a, b) and an estimate of its average life were also calculated to be able to assess its relevance. The average life for the previous article was estimated using two indicators: first by calculating the year in which $50 \%$ of the citations were reached and secondly by applying an equivalent to the finance duration method. Accordingly, 2011 was estimated as the year when 50\% of the citations were achieved for an average article life of 10 years (Table 1).

The economic evaluation of the MAGNA Plan up to 2018 has allowed the authors to study both the evolution of the additional value generated for the 2004-2018 period under consideration and also, given the great worldwide economic crisis that has occurred, if this depression could lead to doubts about any of the previous 2005 results. The updating of the 2005 monetary valuation up to 2018 was calculated by very simply adding the additional value generated for the projects developed by companies between 2004 and 2018. Additionally to the analysis of this significant new period, the substantial new developments in the IGME geological map distribution channels, by starting to make the digitalised MAGNA Plan 1:50,000 scale sheets available globally and free of charge in 2007havebeen considered. It was initially thought that this free electronic downloading might have had very critical implications through its effect on both the simultaneous wide-spreading and dissemination of the IGME cartography. By wide-spreading we understand the downloading of cartographic information by a general public with enough culture to be interested in these mapping products, but without any direct economic purposes in mind, allowing additionally for the understanding of the motivation and importance of these user profile types. These downloads are just for the illustration of its users, for their general curiosity or at most for the development of any open-air leisure activities. The remaining downloads that can be called diffusion, differ from the previous ones in that the uses for this cartographic information are either for scientific or technical purposes. The open, free and permanent access to the results of scientific and technical research, as is the case for the MAGNA Plan maps, is an important aspect of the dissemination of scientific knowledge. Dissemination commonly refers to the provision of scientific knowledge to a particular and specialized audience, made by a specific group of qualified and competent peers or experts in the scientific or technical community of a given field (Ramírez Martínez et al., 2012), an aspect covered specifically in the case of the MAGNA Plan sheets due to their demand by companies, universities, researchers and other professional users.

Table 2 shows the evolution, from 2007 to 2018, of the online consultations of the MAGNA maps, using the only indicator currently provided by the system: the number of unique page views and the average time per page spent by user for each specific query. Unfortunately, the currently available records at IGME, neither allow for the knowledge of the type of use for the maps, nor for company or individual entry types and do not even allow us to know whether connection times are just for map downloading or for alternative long-lasting online consultations. Therefore, with the currently existing data it is not possible to discriminate if the online distribution responds to real information dissemination for scientific and technical purposes for productive users or just for information wide-spreading for individual curiosity or leisure objectives. What is really 
Alfonso Herrero de Egaña, et al., 2020. Análisis del impacto y actualización hasta 2018... Boletín Geológico y Minero, 131 (4): $817-829$

accountable is that the new additional on-line distribution of maps has generated a substantial decrease in paper copy sales from 2011 (the year when sales data for MAGNA paper sheets were available again) to 2018, as the latter are obviously being replaced by the growing on-line free downloading through the IGME web. This declining evolution on paper of MAGNA Plan geological sheets sales is shown in Table 3.

In the absence of any discriminating data for the MAGNA Plan sheet sales and downloads for economic purposes for the new studied period 2004-2018, an estimate of its annual uses by companies has been made by analysing its correlation with the evolution for this period of the Spanish construction industry (which includes building, public and private construction and other activities). Figure 2 shows the statistically significant correlation between the sales of the MAGNA maps over time and the natural logarithm of the 2004 to 2018 evolution of the Spanish construction industry.

\section{Discussion and results}

By using the relationship between the Spanish construction industry and the uses of companies of MAGNA Plan cartography for the previous 1972-2003 time series on map units used or sold to companies and on the accumulated sales to companies, built by García-Cortés et al. $(2005 a, b)$ it was possible to estimate and complete the results up to 2018 (Tables 4, 5 and 6). The authors were then able to quantify the monetary value equivalent to the aggregate social value of the MAGNA Plan, using for consistency both the same measurement variables and the methodology developed for the previous study. Table 7 shows the new estimates for the economic value for 2018 of the MAGNA Plan for all the different types of projects in which its maps have been used. A maximum valuation of about 7,901.62 million euros, a minimum valuation of 2,969.08 million euros, 606.82 million euros by company users willing to pay and 5,266.31 million euros as replacement costs for the equivalent geological investment required. These figures compare very favourably with all the investments required by the MAGNA Plan estimated and updated up to 2018 at 165.02 million euros. Additionally and by comparing with the equivalent valuations for 2003 (Table 8), we can conclude that because of the deep economic crisis suffered during the new study period, the monetary value equivalent to the social value for the MAGNA Plan has obviously increased but not as originally expected. The new estimates for the uses of the MAGNA maps account for only 81.7\% in 2014 and for $87.4 \%$ in 2018 of the highest 2008 sales to companies. Logically some positive additional results are also obtained by comparing Tables 9 and 10 on generated added values, multiple on investments and break-even points for 2018 vs. 2003.

Whilst it is accepted that the equivalent monetary value of the MAGNA Plan involves a direct transfer of income from the general taxes collected, to the very specific group of companies involved in the development of infrastructure projects, which are the ones which have benefited from this geological survey; it is also very true that by reducing the costs of these developments they have had a positive impact on millions of people in a very wide general public, who have benefited all over the country from these new civil works, groundwater, housing construction, agriculture, mining, etc. Therefore we can summarize that the benefits calculated as the added value to the Spanish society of the MAGNA Plan geological 1:50,000 scale survey have really contributed one way or another to the well-being of the great majority of people living in Spain.

\section{Introducción}

En García-Cortés et al. (2005a, b) se analizó la valoración económica y social de las hojas geológicas de la serie MAGNA del Instituto Geológico y Minero de España (en adelante IGME), en base a una amplia encuesta dirigida a un gran número de empresas usuarias de estas cartografías, en la que se solicitaba información sobre el valor atribuido por estas empresas a los mapas geológicos, la disposición al pago por estos productos y el importe de las inversiones en investiga- ción geológica sustitutiva de estos mapas en caso de su inexistencia El seguimiento del impacto científico de estas publicaciones anteriores, mostró un elevado número de citas, con un número significativo de ellas procedentes de fuentes de gran credibilidad. De las dieciocho citas recogidas cabe destacar las siguientes: Comité de l'Académie pour les Applications de la Science (2007), Krenmayr (2009), Duke (2010), Andrews (2011), Berg et al. (2011), Morten (2011), Correia Romão y Arriaga e Cunha (2012), Satkunas (2013), Asch (2014), World Bank (2014), Häggquist and Söder- 
holm (2015), Kruse et al. (2017), siendo todas las citas mencionadas de revistas o trabajos internacionales sobre geología y cartografía geológica.

Los resultados de este análisis, junto a la novedad que supuso la introducción en el año 2007de la disponibilidad electrónica gratuita de dichas hojas MAGNA, con posterioridad a las dos publicaciones sobre el anterior estudio, animó a los autores de este articulo a actualizar la valoración económica de dicho Plan MAGNA. Estos nuevos análisis habrían de incluir, obviamente, tanto el incremento potencial en la divulgación de las hojas MAGNA generado por la facilidad de acceso debida a su descarga electrónica hasta 2018, como el impacto económico generado por sus utilizaciones hasta esa fecha, para permitir actualizar su valoración económica.

Este artículo se divide en seis apartados: tras esta introducción, se presenta brevemente la metodología adoptada, para posteriormente dedicar un tercer apartado al análisis de la repercusión obtenida por la difusión en el ámbito académico de la evaluación económica del Plan MAGNA realizada por García-Cortés et al. $(2005 \mathrm{a}, \mathrm{b})$; en el cuarto apartado, se explica la construcción de la serie de datos utilizados para la valoración, comentando los datos disponibles, las dificultades encontradas y detallando la relación estadística que vincula el número de hojas vendidas o usadas con la variable causa; en el quinto, se valoran los recursos del Plan MAGNA del IGME a precios de 2018, al objeto de su posterior comparación con la valoración obtenida en las anteriores publicaciones; terminando con un último punto que recoge las conclusiones.

\section{Metodología}

La metodología utilizada en la evaluación económica y social realizada en los trabajos de García-Cortés et al. $(2005 a, b)$ se basó en una gran encuesta realizada a numerosas empresas usuarias de las cartografías geológicas de la Serie MAGNA y en la determinación del número de mapas adquiridos por estas entidades. Los registros actualmente disponibles en el IGME no permiten conocer con la suficiente precisión el tipo de usuario de estas cartografías, porque tan sólo se dispone de datos de las ventas de mapas en papel entre 2011 a 2018. A esto se suma que, desde 2007, se ha producido un cambio tecnológico radical, con la puesta a disposición del público, de manera gratuita en la web del IGME, de dichas cartografías geológicas en formato digital ráster (no vectorial) así como de las memorias e información complementaria en formato pdf. En este estudio no se han considerado las descargas de cartografía vectorial por coherencia con el producto analizado durante el periodo 19722003 (cartografía en soporte papel). Dado que estas descargas vectoriales no son gratuitas, las cifras que arroje la actualización de la evaluación económica del Plan MAGNA que se aborda en este trabajo podrían por tanto considerarse como conservadoras. La metodología para llegar a una valoración precisa está condicionada por los datos disponibles sobre el servidor web de cartografía, que eran únicamente el número de páginas vistas, número de páginas vistas únicas y tiempos de permanencia por usuario, proporcionados por el IGME desde 2007 hasta 2018.

Una página vista hace referencia a la visualización de una página en un sitio web que el código de seguimiento de Google Analytics está controlando. Si un usuario vuelve a cargar la página después de que se haya cargado completamente, esto contará como una página vista adicional. Si un usuario navega a una página diferente y más tarde vuelve a la página original, también se registrará una segunda página vista. Una página vista única agrupa las páginas vistas que genera el mismo usuario durante la misma sesión y representa el número de sesiones durante las cuales se ha visto esa página al menos una vez.

En el intento de estimar con la adecuada precisión la utilización de las hojas MAGNA, partiendo de las estadísticas disponibles, nos encontramos con dificultades insalvables, específicamente sobre el uso dado a esos recursos de libre disposición por parte de los usuarios. Este problema incitó a los autores de este trabajo a desarrollar una investigación comparativa de los impactos obtenidos internacionalmente por la divulgación electrónica gratuita de la información científica, asimilando el valor obtenido por la divulgación de artículos científicos de forma gratuita al valor generado por las hojas MAGNA.

En primera instancia se analizó la posible valoración económica a través de estudios internacionales de la repercusión de la disposición gratuita y en línea de los recursos científicos. De este análisis destacan los trabajos de Nicholas et al. (2009), que revelan el gran impacto que han tenido los buscadores en el incremento del uso de los recursos de libre acceso. Aunque las estadísticas dicen mucho sobre el uso que se ha hecho de los recursos, nada pueden decir sobre quién los ha usado y para qué. Si bien el número de citas obtenidas por recursos científicos de descarga gratuita es la medida de su utilidad y por tanto del valor para sus usuarios, al ser las citas en revistas académicas un indicador muy fiable sobre su calidad y utilidad, se observa que, aunque exista un gran aumento del número de usuarios de estos artículos, esto ha tenido escasa influencia en el incremento en el número de citas en el año inmediatamente posterior a su publicación. Davis et al. (2003) y 
Davis (2010 y 2011) abundan en la opinión anterior. Las investigaciones realizadas detectaban un incremento de solamente un $1 \%$ en el número de citas de los artículos con libre acceso virtual frente a los artículos de revistas por subscripción, mostrando que este libre acceso tiene poca influencia entre el mundo científico. Únicamente los artículos a disposición del público con libre acceso en la página web o recurso similar propiedad del autor del artículo, tuvieron un incremento en citas del $11 \%$, aunque este dato no pudo considerarse estadísticamente significativo al tratarse únicamente de un $2 \%$ de los datos. Entre las conclusiones obtenidas por los mencionados autores, hay que destacar que en su opinión los verdaderos beneficiados por el libre acceso son los usuarios que están fuera de la comunidad científica. El público no especializado es el que se ha beneficiado del acceso gratuito, aunque se desconoce el grado y extensión de la difusión de ese conocimiento científico en las redes sociales. Aplicado al presente estudio, este análisis permitiría atribuir los incrementos y decrementos porcentuales de las páginas vistas únicas relacionadas con el Plan MAGNA al sector de otros usuarios distintos a las empresas sin poder precisar su valor.

McCabe y Snyder (2015) realizan un estudio entre las más importantes plataformas de divulgación del conocimiento científico, Elsevier's, ScienceDirect, EconLit, JSTOR, Ebsco y ProQuest y buscadores como Google. No encuentran un impacto significativo en las citas de los artículos de libre acceso electrónico que contienen las plataformas mencionadas, salvo en el caso de JSTOR, que lo atribuyen a que esta última plataforma permite tener acceso a artículos antiguos de gran calidad que son difíciles de encontrar físicamente. Aunque Ebsco y ProQuest también los proporcionan ahora, el hecho de que JSTOR tomase la iniciativa en este campo le ha dado una ventaja competitiva.

Existen otros estudios, como el de Archer y CrosweIl (1989) dedicado al análisis del libre acceso a recursos geográficos y que abunda en la idea del beneficio para el ciudadano, pero que no aporta aspectos cuantitativos interesantes para nuestro estudio. Se ha consultado también un estudio sobre los efectos del acceso en línea a artículos y su efecto en la versión escrita (Vaughan, 2003) que confirma el descenso en las versiones impresas de las revistas. Esto es compatible con el efecto detectado en el IGME de descenso en las ventas en papel de Hojas MAGNA.

Los resultados son enormemente concluyentes en dos aspectos principalmente:

- La divulgación electrónica gratuita aumenta de manera muy importante el número de usuarios que acceden a dichos artículos científicos, reduciéndose asimismo la utilización de los documentos impresos.
- Sin embargo, este hecho no ha producido ningún cambio significativo en el número de citas obtenidas en el mundo científico por dichos artículos.

Asimilando los resultados de la investigación internacional sobre los recursos científicos de libre disposición a los datos de páginas únicas vistas y tiempo de permanencia, se podría afirmar que el incremento de usuarios de las hojas MAGNA viene dado por el sustancial aumento de consultas bibliográficas breves que obviamente en su mayoría no prosperan posteriormente y que, por tanto, no podemos asegurar que generen algún valor económico. Esto se ha podido comprobar, ya que más de $80 \%$ de los accesos a las hojas MAGNA tienen una duración inferior a 0,5 min. Es razonable deducir, por tanto, que estos accesos, que obviamente no prosperan posteriormente, no generan ningún valor económico, coincidiendo con las conclusiones extraídas principalmente de los mencionados estudios de Davis et al. (2003) y Davis (2010 y 2011) sobre el valor económico generado por la gratuidad y facilidad de acceso a los recursos científicos.

Gracias a la investigación realizada se pudo asegurar el escaso o nulo valor económico del uso gratuito de los recursos por parte de los usuarios que no utilizan los recursos para uso profesional. Partiendo por tanto de que la utilización con valor económico real no se veía afectada por la facilidad que supone la disponibilidad electrónica gratuita de las Hojas MAGNA, se decidió estudiar si era posible encontrar una forma de estimar los valores de ventas a empresas para el período de 2004 hasta 2018. Por ello era necesario buscar alguna variable que hubiera tenido relación durante el período de 1972 hasta 2003 con las ventas de hojas a empresas. Se pensó entonces en la evolución de los sectores económicos principales usuarios y muy en particular de la construcción, que incluye edificación, obra civil privada o pública y otras actividades. Se trataba de determinar si existía una relación estadística contrastada entre el número de hojas vendidas por el IGME y la evolución del sector de la construcción (incluida la obra civil) durante los años 1972 a 2003. La hipótesis inicial que avalaba el estudio de esta relación se encontraba en los datos de García-Cortés et al. (2005a), págs. 295 y 296, en los que se establecía una relación de usuarios proveniente de las respuestas al cuestionario cuyas actividades profesionales tenían impacto en la construcción, ya fuera en la edificación o en obra civil. El hecho de que dicho sector sea uno de los que más ha sufrido los efectos de la crisis de manera más intensa añade la virtud de generar adicionalmente cierta tranquilidad sobre la prudencia de dichas estimaciones. Para poder realizar el análisis era necesario disponer de una serie de datos uniforme de la evolución del sector de la construcción para ese pe- 


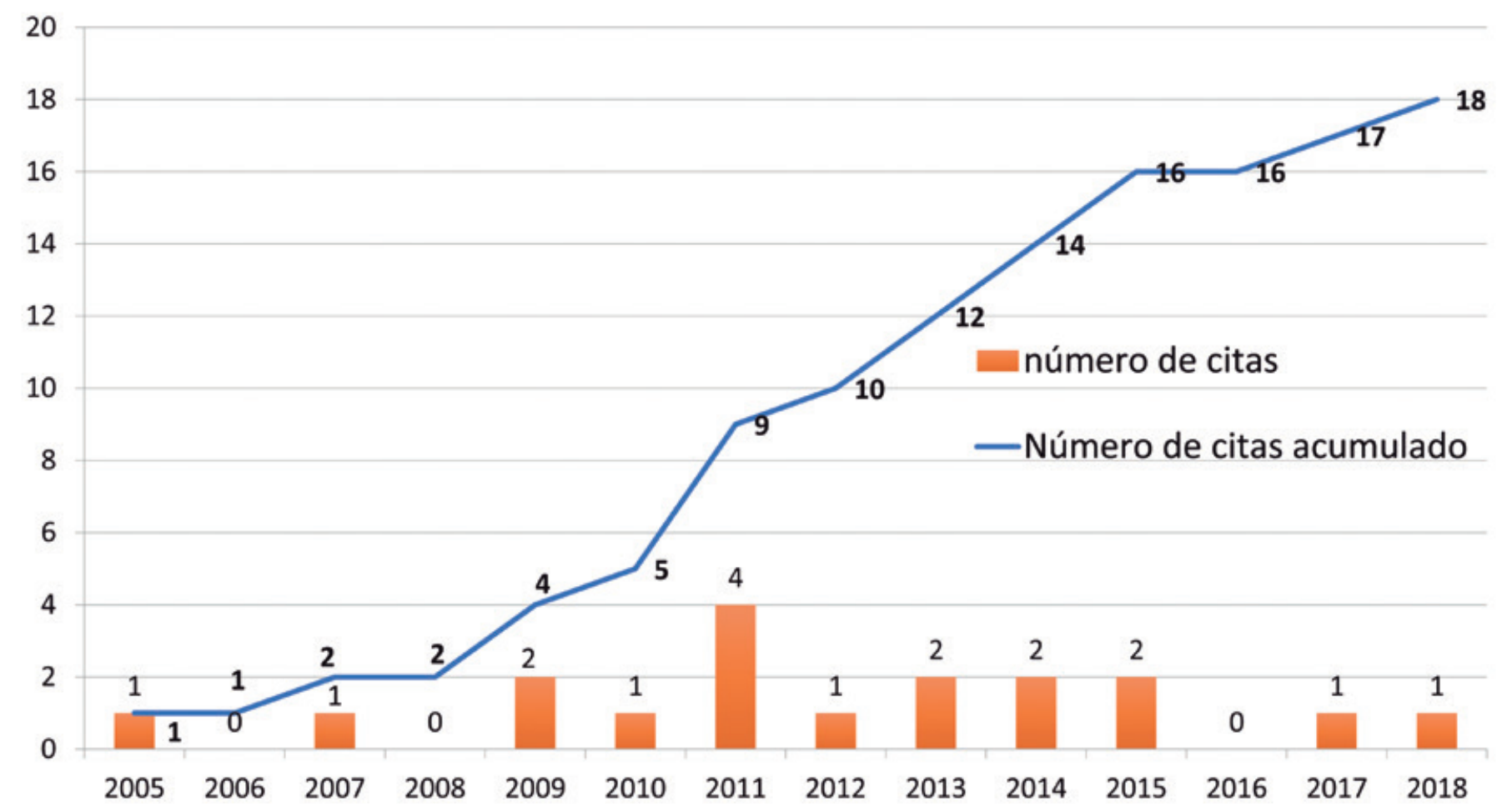

Figura 1. Evolución del número de citas en el período 2005-2018.

Figure 1. Evolution of the number of citations for the 2005-2018 period.

ríodo. Consultados los servicios del INE y la web de ese Instituto se pudo comprobar que no existían ese tipo de datos homogéneos para el período considerado. Tampoco existían esos datos en poder de las asociaciones de empresas del sector. A través de Internet se logró encontrar unos datos (Prados de la Escosura, 2019) para el periodo $1850-2018$ que podrían servir a dicho propósito; este autor había realizado la reconstrucción de series macroeconómicas de largo plazo que permitían situar a España en el contexto de una óptica internacional comparada. Si se demostraba la existencia de una relación estadística entre las ventas de hojas MAGNA y la evolución del sector español de la construcción y obra pública para el periodo 19722003, quedaba solventado el problema.

El estudio estadístico detallado de esta relación se realiza en el apartado de construcción de la serie temporal de datos: limitaciones y dificultades.

Como recomendación derivada del análisis realizado en el presente estudio, y con el objeto de facilitar futuros ejercicios de cuantificación del valor económico de los recursos del Plan MAGNA, se considera que sería conveniente solicitar de los usuarios gratuitos de dichas Hojas MAGNA, como contrapartida, una inscripción simple que, pudiendo incluso ser anónima, especificase como mínimo su actividad económica y la naturaleza de la utilización pretendida de la Hoja MAGNA a la que se accede, y que podría ser al efecto de gran utilidad.

\section{Impacto del estudio publicado de la evaluación social del Plan MAGNA en el ámbito académico}

Los artículos publicados por García-Cortés et al. (2005a, b), se han convertido en artículos de referencia internacional en relevantes revistas académicas en lo todo lo que se refiere a la valoración del impacto económico de los mapas geológicos puestos a disposición del público. En el presente trabajo se ha considerado adecuado realizar una medición del impacto de la difusión del método de valoración monetaria equivalente al valor social propuestos en ambos trabajos en las revistas de carácter científico. En primer lugar, se analizan las citas recibidas por las dos publicaciones, mostrándose su distribución en el tiempo en la figura 1.

Las dos publicaciones han recibido un total $18 \mathrm{ci}-$ tas a lo largo del período 2005-18. Puede observarse además que el número de citas crece a partir del año 2010 , estando el $86 \%$ de las citas concentrado en los años finales del período contemplado. 
Por esta razón se considera que el número de citas debería ser complementado con otro dato que nos permita saber la vida media de esas citas. Dos artículos con el mismo número de citas pueden ser de índole muy diversa: uno puede haber sido citado 15 o 1.500 veces el primer año y no haber sido citado nunca más; si en el otro el número de citas se incrementa a lo largo del tiempo quiere decir que la vigencia de este artículo se ha mantenido en el tiempo. Para estimar la vida media de un artículo se pueden usar dos indicadores; el primero es el cálculo del año en el que se alcanza el $50 \%$ de las citas; el segundo corresponde a una medida asimilable a la variable financiera duración, obtenida de multiplicar el número de citas recibidas en el año por el orden del año en la serie. Los resultados obtenidos con estos dos análisis se muestran en la Tabla 1.

EI $50 \%$ de las citas se alcanza en durante año 2011, dato que ya da una buena idea de cuál es el valor de la vida media de las citas, pero consideramos que es mejor utilizar el segundo método, que con un único número de 10,06 años nos muestra la vida media, como se aprecia en la Tabla 1.

No se utilizan otros índices conocidos como el índice $\mathrm{h}$ de Hirsch, o el índice $\mathrm{i}-10$ porque, aun siendo adecuados, tratan de medir el impacto del trabajo de un investigador en el ámbito académico por el conjunto de sus artículos y no por el de uno en concreto.

\section{Construcción de la serie temporal de datos: limita- ciones y dificultades}

La vida media calculada de citas a los dos artículos, ligeramente superior a 10 años, confirma la oportunidad de la actualización del valor económico del Plan MAGNA abordada en este trabajo, desde el año 2004 hasta el año 2018. A esto se une, como incentivo adicional, la necesidad de tener en cuenta el impacto que durante este periodo ha tenido la introducción en 2007 por el IGME de la opción de descarga electrónica gratuita para las hojas MAGNA.

La construcción de la serie de datos de 2004 hasta 2018, como se ha explicado en el apartado de metodología, es dificultosa porque no se disponen de datos ciertos de ventas a empresas y se tienen que realizar estimaciones. Los únicos datos disponibles lo son a partir de 2007 y corresponden a las páginas vistas únicas y tiempos de permanencia en la página, facilitados por el IGME. Esta parte ha supuesto el mayor reto, debido a la dificultad de valorar un cambio tecnológico importante en la difusión de las hojas, con la dificultad añadida de ser éstas gratuitas y de no disponer de datos específicos sobre el uso asignado ni el origen de las descargas (más concretamente, si fueron realizadas por particulares o por empresas). Los datos disponibles se presentan en la Tabla 2.

Los registros actualmente disponibles en el IGME no permiten conocer el tipo de usuario de esas páginas, o si las entradas en el sistema corresponden a empresas o a particulares. Tampoco es posible inferir si el tiempo de permanencia es debido a la descarga o al trabajo de consulta en línea. Además de los datos del acceso gratuito en línea, se dispone de las ventas en papel desde 2011 a 2018 (Tabla 3), que solamente permiten constatar la disminución de las ventas en papel. Aunque parece que existe una ligera subida de ventas en papel en 2016, no es tal, sino que obedece a que ese año se firmó un Convenio de colaboración con la Sociedad Geológica de España para suministrar gratuitamente, a partir de entonces, cartografía geológica para el evento "Geolodía" que se organiza cada año el segundo fin de semana de mayo.

Un análisis de los datos disponibles de las descargas, reducidos únicamente al número de páginas vistas únicas y al tiempo de permanencia correspondiente en cada una, permitió desechar su utilidad, por insuficientes y por las razones mencionadas en el

\begin{tabular}{|c|c|c|c|c|c|c|c|c|c|c|c|c|c|c|}
\hline AÑO & 2005 & 2006 & 2007 & 2008 & 2009 & 2010 & 2011 & 2012 & 2013 & 2014 & 2015 & 2016 & 2017 & 2018 \\
\hline Acumulado & 1 & 1 & 2 & 2 & 4 & 5 & 9 & 10 & 12 & 14 & 16 & 16 & 17 & 18 \\
\hline Citas & 1 & 0 & 1 & 0 & 2 & 1 & 4 & 1 & 2 & 2 & 2 & 0 & 1 & 1 \\
\hline Producto & 1 & 0 & 2 & 0 & 8 & 5 & 36 & 10 & 24 & 28 & 32 & 0 & 17 & 18 \\
\hline Suma Producto & 181 & & & & & & & & & & & & & \\
\hline Suma & 18 & & & & & & & & & & & & & \\
\hline
\end{tabular}

Tabla 1. Estimación de la vida media del artículo y evolución del porcentaje de citas acumuladas con respecto al total de citas recibidas, durante el periodo 2005-2018.

Table 1. Average article life estimate and percentage evolution of cumulative citations over total citations for the 2005-2018 period. 
Alfonso Herrero de Egaña, et al., 2020. Análisis del impacto y actualización hasta 2018... Boletín Geológico y Minero, 131 (4): $817-829$

\begin{tabular}{|r|r|r|r|r|r|}
\hline AÑO & Número de PVU & $\begin{array}{c}\text { Incremento } \\
\text { Anual }\end{array}$ & $\begin{array}{c}\text { Incremento } \\
\text { Acumulado }\end{array}$ & $\begin{array}{c}\text { Tiempo promedio por } \\
\text { página (segundos) }\end{array}$ & $\begin{array}{c}\text { Tiempo total acumulado } \\
\text { (horas/año) }\end{array}$ \\
\hline 2007 & 189.419 & $-167,18$ & $7.166,23$ \\
\hline 2008 & 167.926 & $-11,35 \%$ & $-11,35 \%$ & 112,89 & $5.266,09$ \\
\hline 2009 & 167.939 & $0,01 \%$ & $-11,34 \%$ & 99,20 & $4.627,72$ \\
\hline 2010 & 196.221 & $16,84 \%$ & $3,59 \%$ & 99,07 & $5.399,64$ \\
\hline 2011 & 226.614 & $15,49 \%$ & $19,64 \%$ & 106,21 & $6.685,79$ \\
\hline 2012 & 260.677 & $15,03 \%$ & $37,62 \%$ & 128,20 & $9.283,17$ \\
\hline 2013 & 288.688 & $10,75 \%$ & $52,41 \%$ & 124,82 & $10.009,69$ \\
\hline 2014 & 268.307 & $-7,06 \%$ & $41,65 \%$ & 129,96 & $9.685,86$ \\
\hline 2015 & 309.907 & $15,50 \%$ & $63,61 \%$ & 122,26 & $10.524,65$ \\
\hline 2016 & 136.699 & $-55,89 \%$ & $-27,83 \%$ & 111,53 & $4.235,10$ \\
\hline 2017 & 259.425 & $89,78 \%$ & $36,96 \%$ & 109,39 & \\
\hline 2018 & 335.843 & $29,46 \%$ & $77,30 \%$ & 102,56 & \\
\hline
\end{tabular}

Tabla 2. Datos de páginas vistas únicas (PVU) y tiempos promedio de permanencia de los usuarios.

Table 2. Number of unique page views and average time on page per user.

\begin{tabular}{|c|c|}
\hline Año & Ventas en papel \\
\hline 2011 & 4.114 \\
\hline 2012 & 1.421 \\
\hline 2013 & 2.697 \\
\hline 2014 & 1.272 \\
\hline 2015 & 1.089 \\
\hline 2016 & 1.394 \\
\hline 2017 & 1.310 \\
\hline 2018 & 1.245 \\
\hline
\end{tabular}

se realizó una regresión o ajuste lineal tratando de aproximar la relación de dependencia entre la variable dependiente (el número de ventas) y la posible variable independiente (el nivel de actividad del sector de la construcción desde 1972 hasta 2003).

La relación estadística entre Construcción y las ventas del IGME a empresas es significativa:

Ventas Hojas IGME $=\beta_{0}+\beta_{1}$ Construcción $_{t}+\varepsilon_{t}$ Ventas Hojas IGME $=2473,90+0,1 \cdot$ Construcción ${ }_{t}+\varepsilon_{t}$ $(6,64) \quad(9,47)$

Pero se observó que si la línea de tendencia fuera logarítmica se incrementaba sustancialmente el coe-

Tabla 3. Datos de ventas en papel de hojas MAGNA entre 2011 y 2018 (Fuente: Servicio de Publicaciones del IGME)

Table 3. Sales data of MAGNA paper sheets for the 2011-2018 period (Source: IGME's Publications Service).

apartado metodología, que descartan la aportación de valor en el sector de los usuarios no profesionales. El estudio se centró entonces en actualizar el valor generado para el sector de usuarios profesionales.

Por tanto, los datos disponibles que podían ser objeto de análisis eran únicamente el número de hojas editadas, el número de hojas vendidas a empresas y la inversión realizada para su elaboración hasta 2003, publicados con anterioridad (Tabla 4) por García-Cortés et al. (2005a, b) y los correspondientes desde 2004 hasta 2018 (Tabla 5), facilitados por el IGME. Por esta razón se decidió complementar el estudio con los datos sobre el sector de la construcción-obra pública para el periodo 1972 a 2003, contenidos en el trabajo de Prados de la Escosura (2019), mencionado en apartado de metodología, y con el análisis de la relación entre el número de hojas vendidas y el nivel de actividad del mencionado sector. En definitiva, ficiente de correlación. Tomando el logaritmo natural de la variable Construcción y estimando la relación lineal por Mínimos Cuadrados Ordinarios obtenemos la línea de regresión reflejada en la Figura 2.

$$
\begin{gathered}
\text { Ventas Hojas IGME } E_{t}=\beta_{o}+\beta_{1} \text { LnConstrucción }_{t}+\varepsilon_{t} \\
\text { Ventas Hojas IGME } E_{t}=-16.960,07+ \\
2.283,75 \cdot \text { LnConstrucción }_{t}+\varepsilon_{t} \\
(-27,31) \quad(35,88)
\end{gathered}
$$

Con este modelo se ha construido la serie temporal que se muestra en la Tabla 6, en la que es interesante destacar la reducción calculada superior al $18 \%$ en la utilización de hojas MAGNA en el 2014, el peor año de la crisis, frente a 2008, el año de mayores ventas. Es asimismo de interés indicar que la estimación correspondiente a los usos de hojas en 2018 todavía refleja una reducción superior al $12 \%$ frente a 2008.

Otra de las variables de posible influencia en el número de hojas utilizadas o vendidas, que fue asimismo analizada, es la serie del número de hojas MAGNA disponibles publicadas por el IGME. Se 


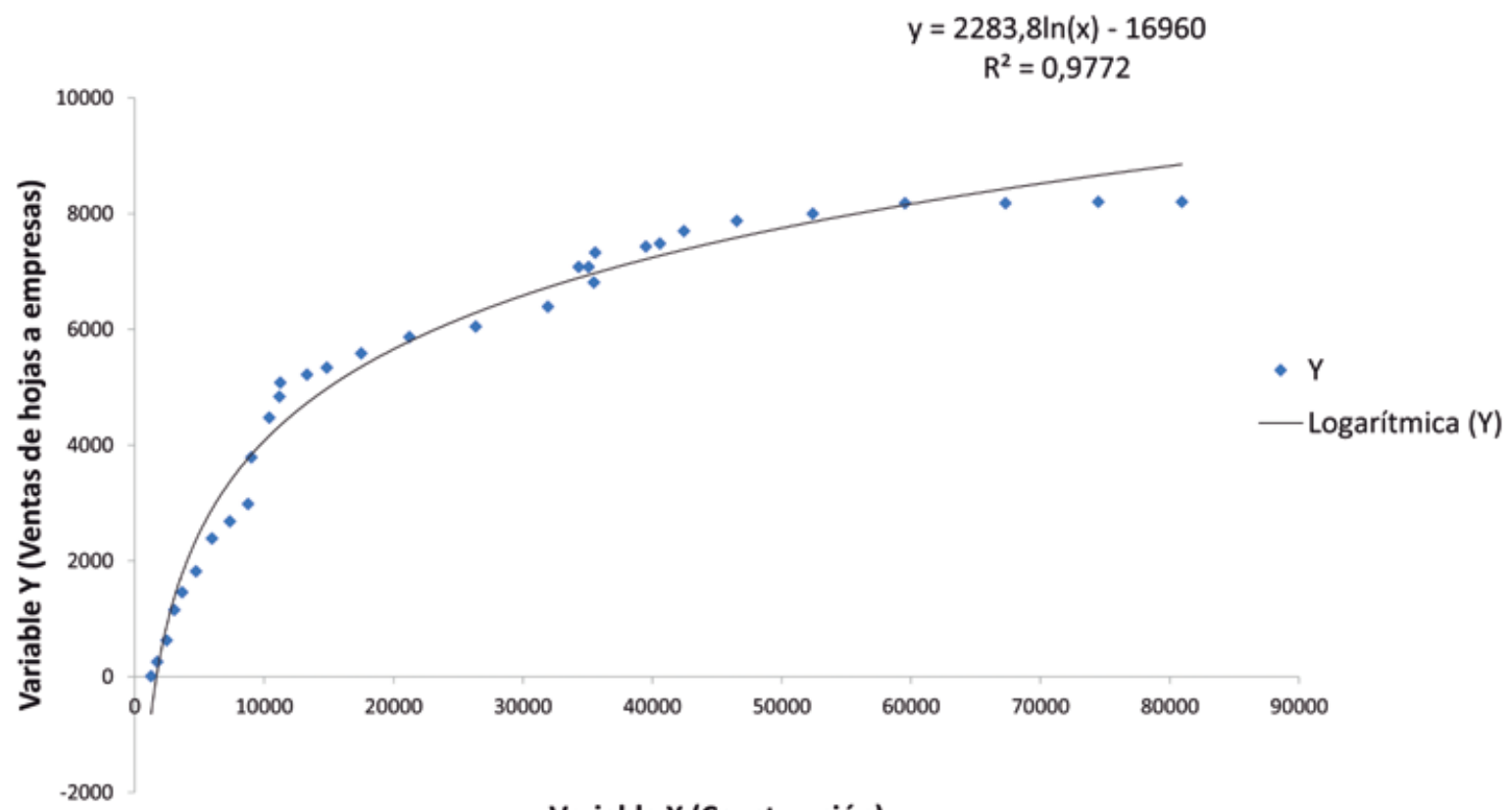

Figura 2. Correlación entre las ventas de hojas MAGNA a empresas y la evolución del sector de la construcción-obra pública.

Figure 2. Correlation between the sales of the MAGNA sheets to companies and the evolution of the Spanish private and public construction industry.

hizo un análisis complementario manteniendo el inicial nivel de actividad del sector construcción como factor determinante de la cantidad de hojas usadas, pero introduciendo la posible influencia del número de hojas disponibles. Sin embargo, hubo que descartar este análisis, debido a la menor significación estadística obtenida a causa de la distorsión generada por cierto grado de colinealidad entre ambas variables independientes, frente a la relación exclusivamente lineal calculada anteriormente con el logaritmo natural de la evolución del sector español de la construcción.

\section{Cuantificación del valor monetario equivalente al va- lor social agregado del Plan MAGNA}

Con los datos acumulados en este estudio se ha abordado una actualización del valor monetario equivalente al valor social para proyectos de las hojas del Plan MAGNA. Para ello hay que complementar el cálculo ya realizado desde 1972 hasta 2003 en García-Cortés et al. (2005a, b), con los cálculos desde 2004 hasta 2018. En todos los casos se distinguirán cuatro valores: un valor mínimo, un valor de disposición de pago, un valor máximo y la estimación de coste/valor de la inversión geológica sustitutiva, que mide el ahorro medio por hoja. Los datos del valor máximo, mínimo, inversión geológica sustitutiva y disposición de pago fueron calculados mediante el desarrollo de una amplia encuesta de usuarios en el año 2005. La correlación observada $\left(R^{2}=0,9772\right)$ entre la evolución del sector de la construcción y el número de hojas vendidas a empresas, y el significativo valor de los estadísticos $(-27,31)$ y $(35,88)$, permite solucionar la ausencia de datos de ventas a empresas entre 2004 y 2018. Tomando por tanto el último dato para 2018 de la serie histórica construida de ventas de hojas MAGNA acumuladas, y aplicando los precios equivalentes actualizados a 2018 obtenidos en la encuesta de usuarios de 2005 citada, se obtiene la valoración económica a 2018 que se muestra en la Tabla 7. Los datos correspondientes hasta 2003 actualizados a valores de 2018 son los indicados en la siguiente Tabla 8.

Si tenemos en consideración que la inversión realizada por el IGME hasta 2003 en el Plan MAGNA fue de160,04 millones de euros de 2018, con un coste medio equivalente por hoja de 173.202€ tendríamos los datos equivalentes a 2003 (García-Cortés et al., 2005a, b) actualizados a 2018 que se muestran en la Tabla 9. 


\begin{tabular}{|c|c|c|c|}
\hline AÑ OS & $\mathbf{N}^{\mathbf{O}}$ de hojas disponibles en el IGME & $\begin{array}{c}\text { Número de hojas para uso o venta a } \\
\text { empresas }\end{array}$ & $\begin{array}{c}\text { Acumulado para uso } \\
\text { o ventas a empresas }\end{array}$ \\
\hline 1972 & 1 & 9 & 966 \\
\hline 1973 & 29 & 258 & 897 \\
\hline 1974 & 71 & 630 & 2.051 \\
\hline 1975 & 130 & 1.154 & 3.508 \\
\hline 1976 & 164 & 1.456 & 5.328 \\
\hline 1977 & 205 & 1.820 & 7.717 \\
\hline 1978 & 269 & 2.389 & 10.398 \\
\hline 1979 & 302 & 2.682 & 17.382 \\
\hline 1980 & 336 & 2.984 & 21.649 \\
\hline 1981 & 427 & 3.792 & 26.489 \\
\hline 1982 & 504 & 4.476 & 31.568 \\
\hline 1983 & 545 & 4.840 & 36.790 \\
\hline 1984 & 572 & 5.079 & 42.127 \\
\hline 1985 & 588 & 5.221 & 47.712 \\
\hline 1986 & 601 & 5.337 & 53.582 \\
\hline 1987 & 629 & 5.586 & 59.629 \\
\hline 1988 & 661 & 5.870 & 66.023 \\
\hline 1989 & 681 & 6.047 & 72.834 \\
\hline 1990 & 720 & 6.394 & 79.911 \\
\hline 1991 & 767 & 6.811 & 86.988 \\
\hline 1992 & 797 & 7.077 & 94.314 \\
\hline 1993 & 797 & 7.077 & 101.747 \\
\hline 1994 & 825 & 7.326 & 109.233 \\
\hline 1995 & 837 & 7.433 & 116.932 \\
\hline 1996 & 843 & 7.486 & 124.808 \\
\hline 1997 & 867 & 7.699 & 132.809 \\
\hline 1998 & 887 & 7.877 & 140.988 \\
\hline 1999 & 901 & 8.001 & 149.166 \\
\hline 2000 & 921 & 8.178 & 157.371 \\
\hline 2001 & 921 & 8.178 & 165.576 \\
\hline 2002 & 924 & 8.205 & \\
\hline 2003 & 924 & 8.205 & \\
\hline
\end{tabular}

Tabla 4. Número de hojas MAGNA para uso o venta a empresas y acumulado de ventas a empresas. Serie construida $1972-2003$ por García-Cortés et al.(2005a, b).

Table 4. Sales and cumulative sales data of MAGNA sheets to companies for the 1972-2003 period. Time series built by García-Cortés et al. $(2005 a, b)$.

En la que el valor añadido es la diferencia entre el valor total y los citados 160,04 millones de euros de 2018 de coste para el IGME hasta 2003 del proyecto MAGNA. El múltiplo de la inversión es la razón entre el valor total y el coste, y el cálculo del punto muerto la razón entre el coste por hoja y el correspondiente valor máximo, mínimo etc. Se consigue por tanto una estimación mínima del valor añadido generado por el Plan MAGNA hasta 2003 de 1.496,05 millones de euros de 2018.

Para el cálculo de los datos en el período de 2004 hasta 2018 hay que tomar en consideración los datos que se muestran en la Tabla 9, que incluyen tanto los costes de la elaboración de nuevas hojas, así como por las actualizaciones de hojas MAGNA y el correspondiente incremento en el número de hojas publicadas y por tanto disponibles.

Los datos facilitados por el Departamento de Investigación y Prospectiva Geocientífica del IGME cuantifican la totalidad de los costes adicionales incurridos por el IGME en desarrollos y actualizaciones de hojas

\begin{tabular}{|c|c|c|}
\hline AÑOS & Inversión (€) & Hojas publicadas \\
\hline 2004 & 542.554 & 967 \\
\hline 2005 & 593.860 & 979 \\
\hline 2006 & 883.344 & 1.012 \\
\hline 2007 & 254.047 & 1.026 \\
\hline 2008 & 669.881 & 1.040 \\
\hline 2009 & 289.300 & 1.062 \\
\hline 2010 & 30.000 & 1.072 \\
\hline 2011 & 38.279 & 1.072 \\
\hline 2012 & 56.557 & 1.078 \\
\hline 2013 & 152.352 & 1.092 \\
\hline 2014 & 104.127 & 1.099 \\
\hline 2015 & 140.545 & 1.110 \\
\hline 2016 & 122.007 & 1.116 \\
\hline 2017 & 39.368 & 1.116 \\
\hline 2018 & 22.456 & 1.116 \\
\hline
\end{tabular}

Tabla 5. Inversión destinada al Plan MAGNA y número de hojas geológicas publicadas desde 2004 hasta 2018 (Fuente: Departamento de Investigación y Prospectiva Geocientífica, IGME).

Table 5. Investments devoted to the MAGNA Plan and number of geological sheets published from 2004 to2018/Source: Research and Geoscientific Prospective Department, IGME). 
Alfonso Herrero de Egaña, et al., 2020. Análisis del impacto y actualización hasta 2018... Boletín Geológico y Minero, 131 (4): $817-829$

\begin{tabular}{|c|c|c|c|}
\hline AÑOS & $\mathrm{N}^{\circ}$ de hojas disponibles en el IGME & $\begin{array}{c}\text { Número de hojas para uso o venta a } \\
\text { empresas }\end{array}$ & $\begin{array}{c}\text { Acumulado para uso o ventas a } \\
\text { empresas }\end{array}$ \\
\hline 2005 & 979 & 9.310 & 183.916 \\
\hline 2007 & 1.026 & 9.614 & 203.057 \\
\hline 2008 & 1.040 & 9.662 & 212.719 \\
\hline 2009 & 1.062 & 9.499 & 222.218 \\
\hline 2011 & 1.072 & 8.631 & 239.878 \\
\hline 2012 & 1.078 & 8.267 & 248.145 \\
\hline 2013 & 1.092 & 7.917 & 256.062 \\
\hline 2014 & 1.099 & 7.895 & 263.957 \\
\hline 2015 & 1.110 & 8.026 & 271.983 \\
\hline 2016 & 1.116 & 8.142 & 280.125 \\
\hline
\end{tabular}

Tabla 6. Estimación del número anual de hojas MAGNA para uso o venta a empresas y acumulado de ventas a empresas. Serie construida entre 2004-2018 a partir de la evolución del sector de la construcción.

Table 6. Uses/sales and cumulative user/sales estimates of MAGNA sheets to companies. 2004 to 2018 time series built from the evolution of the Spanish construction industry.

MAGNA para el periodo 2004-2018 en 3.938.677 € nominales. Cantidad que, invertida durante cada uno de estos años, hay que normalizar para su comparabilidad mediante el cálculo de la cantidad financieramente equivalente para el año 2018,mediante la utilización de los datos de inflación media desde el año 2004 hasta 2018 (https://es.inflation.eu/tasas-de-inflacion/ espana/inflacion-historica/ipc-inflacion-espana.aspx). Esta inversión se contabiliza, a precios de 2018,en 4.979.611€,lo que reduce el coste total medio por hoja desde $173.201 €$ a $147.865 €$, dadas las menores inversiones destinadas al Plan MAGNA durante el período de 2004 hasta 2018, y que implica un coste total de la inversión realizada por el IGME, en este Plan, de 165,02millones de euros de 2018.Estareducciónen el coste medio por hoja elaborada por el IGME se debe, tanto al incremento en el número de hojas publicadas(parte de cuyos costes de ejecución se habían contabilizado hasta 2003) como a las menores inversiones requeridas por las últimas hojas publicadas, al haber sido elaboradas directamente por personal del IGME, no contabilizándose en dichas inversiones sus correspondientes costes salariales.

En la Tabla 10 se muestra el valor añadido mínimo, el múltiplo de la inversión y el punto muerto calculado para 2018 del Plan MAGNA.

\begin{tabular}{|c|r|r|r|}
\hline Tipo de valor & No Hojas vendidas a empresas & \multicolumn{1}{|c|}{ Valor en $€$} & Valor Total (millones de $€$ ) \\
\hline Máximo & 296.853 & 26.618 & $7.901,63$ \\
\hline Mínimo & 296.853 & 10.002 & $2.969,12$ \\
\hline Disposición de pago & 296.853 & 2.044 & 606,77 \\
\hline Inversión geológica sustitutiva & 296.853 & 17.740 & $5.266,17$ \\
\hline
\end{tabular}

Tabla 7. Valor Económico, para todo tipo de proyectos, de las hojas MAGNA hasta 2018.

Table 7. Economic value to 2018 for all different types of projects of the MAGNA maps.

\begin{tabular}{|c|r|r|r|}
\hline Tipo de valor & $\mathbf{N}^{\circ}$ Hojas vendidas a empresas & \multicolumn{1}{|c|}{ Valor en $€$} & Valor Total (millones de $€$ ) \\
\hline Máximo & 165.576 & 26.618 & $4.407,30$ \\
\hline Mínimo & 165.576 & 10.002 & $1.656,09$ \\
\hline Disposición de pago & 165.576 & 2.044 & 338,44 \\
\hline Inversión geológica sustitutiva & 165.576 & 17.740 & $2.937,32$ \\
\hline
\end{tabular}

Tabla 8. Valor Económico, para todo tipo de proyectos, de las hojas MAGNA hasta 2003, actualizado a 2018.

Table 8. Economic value to 2003 for all different types of projects of the MAGNA maps, updated to 2018. 
Alfonso Herrero de Egaña, et al., 2020. Análisis del impacto y actualización hasta 2018... Boletín Geológico y Minero, 131 (4): $817-829$

\begin{tabular}{|l|r|r|r|r|r|}
\hline \multicolumn{1}{|c|}{$\mathbf{1 9 7 2 - 2 0 0 3}$} & Valor por hoja (€) & $\begin{array}{c}\text { Valor Total } \\
\text { (millones de } € \text { ) }\end{array}$ & $\begin{array}{c}\text { Valor añadido (mil- } \\
\text { lones de } € \text { ) }\end{array}$ & $\begin{array}{c}\text { Múltiplo } \\
\text { inversión }\end{array}$ & Punto muerto (Nº de hojas) \\
\hline Valor Máximo & 26.618 & $4.407,30$ & $4.247,26$ & 27,54 & 6,51 \\
\hline Valor Mínimo & 10.002 & $1.656,09$ & $1.496,05$ & 10,35 & 17,32 \\
\hline Disposición de pago & 2.044 & 338,44 & 178,40 & 2,11 & 84,74 \\
\hline $\begin{array}{l}\text { Inversión geológica susti- } \\
\text { tutiva }\end{array}$ & 17.740 & $2.937,32$ & $2.777,28$ & 18,35 & \\
\hline
\end{tabular}

Tabla 9. Datos financieros del Plan MAGNA en 2003 actualizados a 2018.

Table 9. Financial data of the MAGNA Plan to 2003, updated to 2018.

\begin{tabular}{|c|c|c|c|c|c|}
\hline $1972-2018$ & Por hoja (€) & Total (millones de $€$ ) & $\begin{array}{l}\text { Valor añadido } \\
\text { (millones de } € \text { ) }\end{array}$ & $\begin{array}{l}\text { Múltiplo } \\
\text { inversión }\end{array}$ & Punto muerto ( $\mathrm{N}^{\circ}$ de hojas) \\
\hline Valor Máximo & 26.618 & $7.901,63$ & $7.736,61$ & 47,88 & 5,56 \\
\hline Disposición de pago & 2.044 & 606,77 & 441,75 & 3,68 & 72,34 \\
\hline Inversión geológica sustitutiva & 17.740 & $5.266,17$ & $5.101,15$ & 31,91 & 8,34 \\
\hline
\end{tabular}

Tabla 10. Datos financieros del Plan MAGNA actualizados para 2018.

Table 10. Financial data of the MAGNA plan up to 2018.

\section{Conclusiones}

El anterior estudio de 2005 abordado por García-Cortés y colaboradores, sobre evaluación económica del Plan MAGNA, ha tenido una buena aceptación, como se demuestra por su impacto en publicaciones científicas. Este hecho es una de las causas que ha animado a los autores a actualizar los resultados obtenidos, desde el año 2004 hasta el año 2018.

El valor monetario agregado equivalente al valor social del Plan MAGNA en 2018 ha continuado lógicamente incrementándose desde 2003. La disminución en los múltiplos de inversión y en los puntos muertos de rentabilidad obedece al incremento en el número de hojas disponibles a partir de 2004, parte de ellas ya realizadas pero no publicadas hasta entonces, y a que esas nuevas hojas fueron elaboradas por personal propio del IGME y no por empresas contratistas, con la consiguiente disminución en el coste medio por hoja publicada. En el anterior trabajo se consideraron 924 hojas, mientras que el valor actual se basa en la disponibilidad de 1.116 hojas debido a las nuevas hojas completadas durante el período del estudio. Estos datos han influido en el cálculo de las ventas acumuladas a empresas hasta 2018, que han seguido creciendo, aunque obviamente a un ritmo inferior al del período 1972 a 2003, que se interpreta como resultado de la profunda crisis económica sufrida.

Se confirma el mantenimiento en el tiempo en la utilización de las hojas por parte de los usuarios, lo que, a pesar de no disponer de datos reales sobre el tipo de uso que reciben, permite deducir la vigencia y utilidad de los recursos del Plan MAGNA para el sector empresarial o para proyectos de contenido económico.

El valor monetario equivalente debe entenderse como una transferencia de renta de los impuestos generales recaudados hacia un grupo de personas, que son las que directamente se han beneficiado del Plan. Cuanto más extensa sea la población que ha recibido esa transferencia, mayores serán los beneficios para el conjunto de la sociedad. Aunque las empresas dedicadas a proyectos son las más beneficiadas de manera directa, y su número es reducido, el menor coste de sus actividades ha repercutido indirectamente en millones de personas, en particular, en todas aquellas que se benefician de las actividades de la obra civil, aguas subterráneas, construcción de viviendas, agricultura, minería, etc., pudiéndose considerar que los beneficios del Plan han redundado prácticamente en el conjunto de la sociedad española por la cantidad calculada como valor agregado.

Basándose en esas estimaciones, se ha calculado que el incremento en el valor económico total de las hojas utilizadas por el sector de empresas en el período de 2004 a 2018 es de 3.494,33 millones de euros en el caso de valor máximo, de 1.313,03 millones de euros en el caso de valor mínimo, de 268,33 millones de euros si se toma en consideración la disposición de pago y de 2.328,85 millones de euros considerándose la inversión geológica sustitutiva. 


\section{Agradecimientos}

Los autores agradecen la detallada y constructiva revisión de este trabajo, efectuada por los doctores Roberto Rodríguez Fernández y Alejandro Robador Moreno, que ha redundado en una considerable mejora en la presentación final de los resultados obtenidos.

\section{Referencias}

Andrews, C. 2011. The Role of the State to Provide Geological Information. 10/12/2018. 26 pp. https://web.archive. org/web/20150208062039/http://craigandrewsllc.com/document_download_3.html.

Archer, H. and Croswell, P.L. 1989. Public Access to Geographic Information Systems: An Emerging Legal Issue, Photogrammetric Engineering and Remote Sensing, Vol. 55 (11), 1575-1581

Asch, K. 2014. Geoscience Information in Africa. GIRAF. CAG 25, Dar es Salaam. 62 pp.

Berg, R.C., Mathers, S.J., Kessler, H. y Keefer, D.A. 2011. Synopsis of Current Three-dimensional Geological Mapping and Modeling in Geological Survey Organizations. Illinois State Geological Survey and British Geological Survey, Circular 578, 104pp.

Buela-Casal, G. 2002. Evaluación de la investigación científica: el criterio de la mayoría. El factor de impacto, el factor prestigio y los diez mandamientos para incrementar las citas. Análisis y Modificación de Conducta, 28(119), 455-476.

Comité de I'Académie pour les Applications de la Science. 2007. La Carte Géologique de Wallonie. Académie Royale des Sciences, des Lettres et des Beaux-Arts de Belgique.14 pp.

CorreiaRomão, J.M. y Arriaga e Cunha, T. 2012. Cartografia geológica. Una mais-valia para o desenvolvimento do territorio.GeonovasAssociaçao Portuguesa de GeólogosNo25, 3-17.

Davis, P.M. and Solla, L.R. 2003. An IP-level analyses of usage statistics for electronic journals in chemistry: Making inferences about user behavior. Journal of the American Society for Information Science and Technology, 54(11), 1062-1068.

Davis, P.M., 2010 Access, readership, citations: A randomized controlled trial of open access publishing in scientific journals. Thesis. 163 pp. Cornell University

Davis, P.M. 2011, Open access publishing, article downloads, and citations: Randomized controlled trial. The FASEB Journal, 25 (7), 2129-2134.

Duke, J.M. 2010. Government geosciences to support mineral exploration: public policy rationale and impact. Prospectors and Developers Association of Canada, 72 pp.
García-Cortés, Á., Vivancos J. y Fernández-Gianotti J. 2005a. Evaluación económica y social del Plan MAGNA. Boletín Geológico y Minero, 116 (4), 291-305.

García-Cortés A., Vivancos J. \& Fernández-Gianotti J. 2005b. Economic and social value of the MAGNA Plan. In Economic and social value of the MAGNA Plan. Geological map of Spain at a scale of 1:50.000. Ed. IGME. 29-51

Häggquist, E. y Söderholm, P. 2015. The economic value of geological information: Synthesis and directions for future research. Resources Policy, 43, 91-100.

Krenmayr, H.G. 2009. Strategie und Stand der geologischenLandesaufnahmedurch die Geologische Bundesanstalt in Österreich. Arbeitstagung Geologische Bundesanstalt Leoben, 189-198

Kruse, J.B, Crompvoets, J. and Pearlman, F. 2017. GEOValue: The Socioeconomic Value of Geospatial Information. CRC Press, $332 \mathrm{pp}$.

McCabe and Snyder, 2015. Does Online Availability Increase Citations? Theory and Evidence from a Panel of Economics and Business Journals. Review of Economics and Statistics, 97 (1), 144-165.

Morten, S. 2011. Norskgeo fagligforskning. Nå?. 20/02/19. Geoforskningblogghttps://www.geoforskning.no/blogg/ item/norsk-geofaglig-forskning-na.

Nicholas, D., Rowlands, P.W., Brown, D. and Clark, D. 2009, E-journals: their use, value and impact: A Research Information Network report. April 2009, Research Information Network. 32 pp. 15/04/19. http://www.rin.ac.uk/system/files/attachments/sarah/E-journals-report.pdf

Prados de la Escosura, L. 2019. Contabilidad nacional histórica de España: gasto y producto, 1850-2018. Espacio Investiga. Fundación Rafael del Pino. 17/05/19. https:// espacioinvestiga.org/bbdd-chne/

Satkunas, J. 2013. GeologinisKartografavimas - GeologuPriedermé ir NaudaVisuomenei. GeologijosPazanga2013(2), 7-10.

Ramírez Martínez, D.C., Martínez Ruiz, L.C y Castellanos Domínguez, F. 2012. Divulgación y difusión del conocimiento: las revistas científicas. Bogotá: Universidad Nacional de Colombia. Programa Interdisciplinario de Investigación y Desarrollo en Gestión, Productividad y Competitividad - BioGestión, 184 pp.

Vaughan, K.T.L. 2003. Changing use patterns of print journals in the digital age: Impacts of electronic equivalents on print chemistry journal use. Journal of the Association for Information Science and Technology, 54, (12), 1149-1152.

World Bank. 2014. The African Minerals Geoscience Initiative (AMGI). The "Billion Dollar Map". Technical Report. 20/02/19https://www.tralac.org/images/docs/6687/the-biIlion-dollar-maptechnical-report.pdf.

Recibido: julio 2019

Revisado: noviembre 2019

Aceptado: enero 2020

Publicado: julio 2020 